\title{
Genome-wide characterisation of the Gcn5 histone acetyltransferase in budding yeast during stress adaptation reveals evolutionarily conserved and diverged roles
}

Yongtao Xue-Franzén ${ }^{1,2^{*}}$, Anna Johnsson ${ }^{1,2}$, David Brodin ${ }^{2 \dagger}$, Johan Henriksson ${ }^{1,2 \dagger}$, Thomas R Bürglin ${ }^{1,2}$, Anthony PH Wright ${ }^{1,2}$

\begin{abstract}
Background: Gcn5 is a transcriptional coactivator with histone acetyltransferase activity that is conserved with regard to structure as well as its histone substrates throughout the eukaryotes. Gene regulatory networks within cells are thought to be evolutionarily diverged. The use of evolutionarily divergent yeast species, such as $S$. cerevisiae and S. pombe, which can be studied under similar environmental conditions, provides an opportunity to examine the interface between conserved regulatory components and their cellular applications in different organisms.

Results: We show that Gcn5 is important for a common set of stress responses in evolutionarily diverged yeast species and that the activity of the conserved histone acetyltransferase domain is required. We define a group of $\mathrm{KCl}$ stress response genes in S. cerevisiae that are specifically dependent on Gcn5. Gcn5 is localised to many Gcn5dependent genes including Gcn5 repressed targets such as FLO8. Gcn5 regulates divergent sets of $\mathrm{KCl}$ responsive genes in S. cerevisiae and S. pombe. Genome-wide localization studies showed a tendency for redistribution of $\mathrm{Gcn} 5$ during $\mathrm{KCl}$ stress adaptation in S. cerevisiae from short genes to the transcribed regions of long genes. An analogous redistribution was not observed in S. pombe.
\end{abstract}

Conclusions: $\mathrm{Gcn} 5$ is required for the regulation of divergent sets of $\mathrm{KCl}$ stress-response genes in $\mathrm{S}$. cerevisiae and S. pombe even though it is required a common group of stress responses, including the response to $\mathrm{KCl}$. Genes that are physically associated with Gen5 require its activity for their repression or activation during stress adaptation, providing support for a role of Gcn5 as a corepressor as well as a coactivator. The tendency of Gcn5 to re-localise to the transcribed regions of long genes during $\mathrm{KCl}$ stress adaptation suggests that $\mathrm{Gcn} 5$ plays a specific role in the expression of long genes under adaptive conditions, perhaps by regulating transcriptional elongation as has been seen for Gcn5 in S. pombe. Interestingly an analogous redistribution of Gcn5 is not seen in S. pombe. The study thus provides important new insights in relation to why coregulators like Gcn5 are required for the correct expression of some genes but not others.

\section{Background}

Evolutionary changes frequently involve modifications to gene regulatory programs. Several lines of evidence indicate the importance of transcriptional regulation in evolution. First, comparative studies have shown that

\footnotetext{
* Correspondence: yongtao.xue@sh.se

+ Contributed equally

${ }^{1}$ School of Life Sciences, Södertörn University, Huddinge, SE-141 89, Sweden
}

cis-acting regulatory elements that are binding sites for regulatory transcription factors evolve rapidly [1-3]. Second, the proportion of genes encoding transcription factors is higher in genomes encoding larger proteomes consistent with the disproportionately higher regulatory requirements of more complex organisms [4-7], For example, the nematode Caenorhabditis elegans has about 100 genes encoding homeobox transcription factors [8] compared to 7 such genes in yeast 
(S. cerevisiae) [4,9], while the total proteome in the nematode is less than 4-fold larger than that in yeast. Third, several yeast species whose common ancestor has undergone whole genome duplication have preferentially maintained paralogous pairs of transcription factors during the re-haploidisation process that ensued, suggesting that transcription factors have a higher than average evolutionary potential [4]. Finally, it has been suggested that non-coding RNA, which is much more abundant in complex organisms with large genomes, plays important roles in regulating the transcription levels of genes [10]. More recently it has been suggested that protein interactions between transcription factors and co-regulator proteins they recruit to target genes may also be important for evolutionary potential $[11,12]$. Thus, evolutionary changes in the interactions of transcription factors with DNA binding sites and the co-regulators they recruit would predict that the involvement of co-regulator proteins in different regulatory pathways should be evolutionarily diverged.

Gcn5 is a phylogenetically conserved transcriptional co-regulator that is found throughout the eukaryotes. Gcn5 was the first transcriptional co-regulator protein shown to contain a histone acetyltransferase (HAT) activity [13] and it is the catalytic subunit of several related HAT complexes, notably the SAGA complex [14]. These complexes acetylate lysine residues in chromatin, primarily within the N-terminus of histone $\mathrm{H} 3$ [15]. The subunit composition of the SAGA complex is highly conserved across the large evolutionary distance between S. cerevisiae and S. pombe [16]. The SAGA complex is a direct target for recruitment by transcriptional activators in vitro $[17,18]$ and in vivo [19] and is physically associated to a greater or lesser extent with the promoter and/or transcribed regions of many expressed genes in S. cerevisiae [17] and S. pombe [20]. The HAT domain is the most highly conserved part of Gcn 5 and it has been shown to be inter-changeable between humans and yeast [21]. Gcn5 is thus structurally conserved throughout evolution and appears to function in a conserved fashion by acetylating a conserved set of lysine residues in target proteins. Interestingly, Gcn 5 has also been reported to be important for regulation of stress-response genes in budding and fission yeasts [22,23], suggesting that some physiological roles of Gcn 5 may also have been conserved but this has not been studied systematically.

Yeasts offer a powerful system for comparative studies because highly divergent organisms can be cultured and manipulated under comparable environmental conditions [24]. Our previous studies show a specific role of fission yeast Gcn5 in programming a subset of stress response genes in $S$. pombe [22]. To further understand functional and evolutionary aspects of Gcn5, we here study the equivalent stress responses in the evolutionarily diverged budding yeasts $S$. cerevisiae and $S$. kluyveri. We show that Gcn5 is required for a common set of stress responses in the budding and fission yeasts. We further report results from a genome-wide study of Gcn5's role in the $\mathrm{KCl}$ stress response in S. cerevisiae that is comparable to our previous studies in $S$. pombe. The results reveal interesting novel insights with regard to the function of Gen5 in S. cerevisiae and show that it regulates a different set of stress genes compared to those identified in $S$. pombe. Further, we show that Gcn5 is located throughout the transcribed regions of many $S$. cerevisiae genes as has also been shown for $S$. pombe [20], but that there are also mechanistic differences in Gcn 5 action between the two species. The study provides an interesting view of the interface between aspects of a transcriptional regulator that are highly conserved and their functionally divergent applications in evolutionarily distant species.

\section{Results and Discussion}

Gon 5 is required for several common stress responses in divergent yeast species

To compare the physiological roles of Gcn5 over a large evolutionary interval, we chose to compare the phenotype of gcn5 5 mutants in $S$. cerevisiae and its distant budding yeast relative $S$. kluyveri, using a range of environmental stress conditions, including several conditions where Gcn 5 dependence has previously been demonstrated in S. pombe [22]. First, we replaced the GCN5 gene from wild type $S$. kluyveri with a standard KANMX cassette as described in the Materials and Methods. Fig. 1A shows the result of testing the phenotypes of gcn5 5 mutants of the three yeast species under different environmental-stress conditions. gcn5 $\Delta$ mutants in $S$. pombe are not distinguishable from wild type cells on rich medium as shown previously [22], but they show a mild growth deficiency in $S$. cerevisiae and S. kluyveri. We observed that Gcn 5 was required in $S$. cerevisiae and $S$. kluyveri for adaptation to stresses mediated by $\mathrm{KCl}$ and $\mathrm{CaCl}_{2}$ as previously observed in S. pombe [22]. Gcn5 is also required in all three species for other stress conditions, such as Calcoflour White, $\mathrm{MnCl}_{2}$ and Caffeine. These common stress sensitivity phenotypes suggest a specific phenotypically conserved role for Gcn5 because Gcn5 dependence is not universally observed in the three different species for a range of other stress conditions. These conditions include $\mathrm{LiCl}, \mathrm{MgCl} 2, \mathrm{NaCl}$ and sorbitol for which none of the yeasts require Gcn5 for stress adaptation. Also for ethanol, elevated temperature and SDS, Gcn 5 appears to be differentially important in the different yeast species (see Additional file 1). Quantitative analysis of all measured colony size 


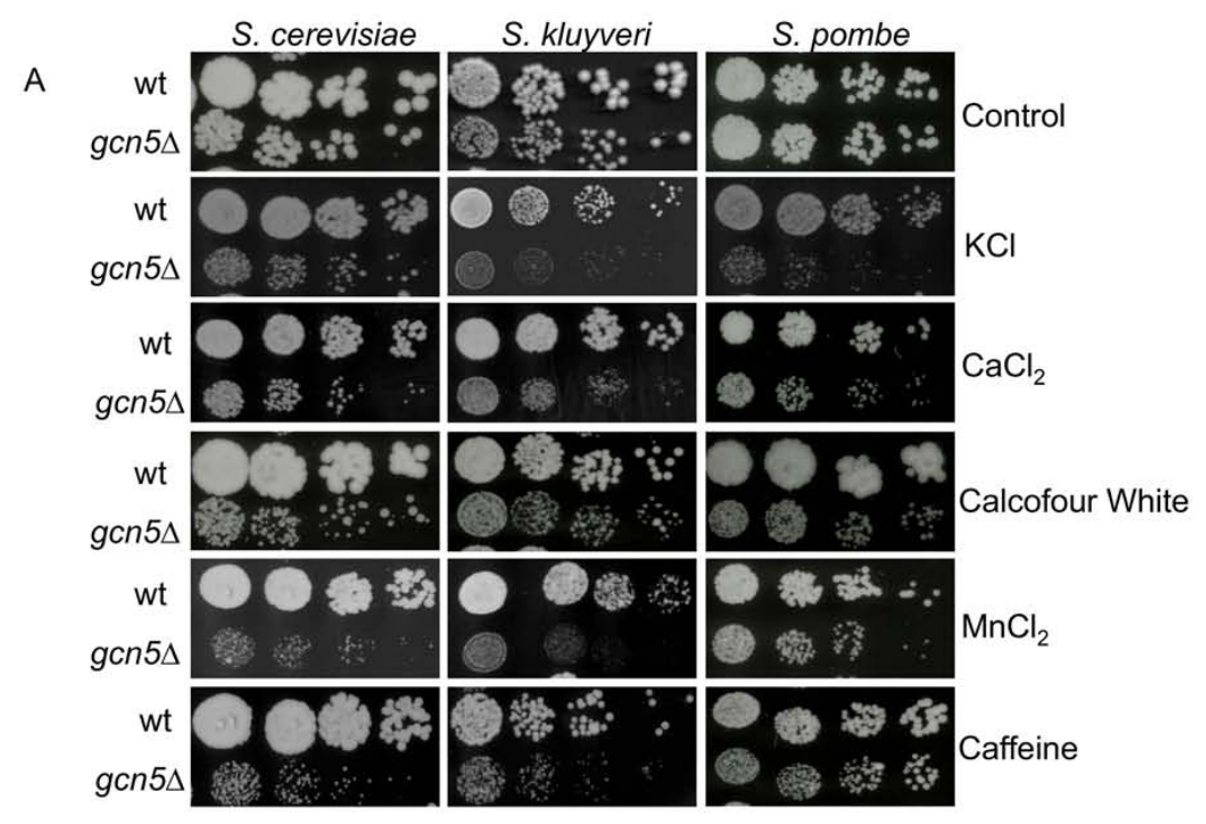

B

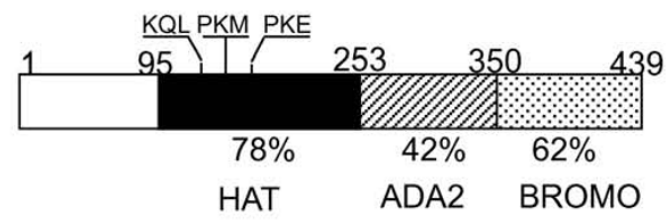

C
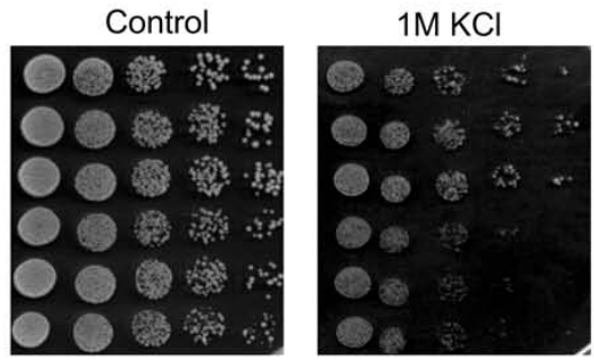

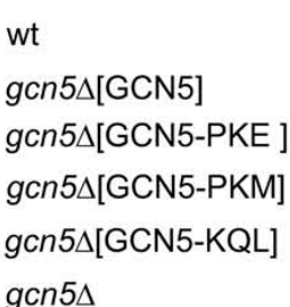

$4 \mathrm{mM} \mathrm{MnCl} 2$

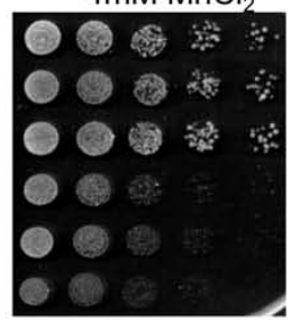

$0.25 \mathrm{M} \mathrm{CaCl}_{2}$

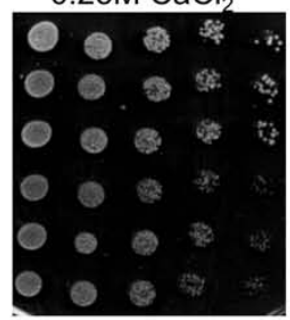

\begin{tabular}{|c|}
\hline$\Delta \Delta[$ GCN5] \\
\hline $5 \Delta[\mathrm{GCN} 5-\mathrm{PKE}]$ \\
\hline$n 5 \Delta[\mathrm{GCN} 5-\mathrm{PKM}]$ \\
\hline $\begin{array}{l}n 5 \Delta[\mathrm{GCN} 5-\mathrm{KQL}] \\
n 5 \Delta\end{array}$ \\
\hline
\end{tabular}

Figure $1 \mathrm{Gcn} 5$ is required for several common stress responses in divergent yeast species and defects in its conserved histone acetyltransferase domain cause stress sensitivity. (A) Gcn5 is required for efficient growth under a common set of environmental stress conditions in evolutionarily divergent yeast species. The figure shows serial dilutions of wild type (wt) or mutant (gcn5 $\Delta$ ) yeast cells spotted on normal growth media (control) or media containing stress inducing levels of $\mathrm{KCl}, \mathrm{CaCl}_{2}$, Calcoflour White, $\mathrm{MnCl}_{2}$ and caffeine. (B) The domain structure of Gen5 showing the highly conserved histone acetyltransferase (HAT). The diagram shows the HAT, the Ada2-interaction domain (ADA2) and the Bromo domain (Bromo) domains defined previously for the $S$. cerevisiae protein [25]. Residue numbers equivalent to domain boundaries are shown. The extent of domain identity between S. cerevisiae and S. pombe is shown (\%). The approximate position of each mutant triple-alanine substitution mutant, starting at residues 126, 129 and 132 respectively, is shown. (C) The HAT activity of Gen5 is required for Gcn5 dependent stress responses. Cell plating assay performed as in Fig. 1A showing S. cerevisiae gcn5 $\Delta$ cells carrying pRS316 plasmids expressing either wild type GCN5 or GCN5 with substitution mutations in the HAT domain as indicated in (B). Wild type (wt) and GCN5 (gcn5 $\triangle$ ) cells were used as controls. 
differences is shown in Additional File 1. The conserved stress conditions thus provide ideal models for studying divergence and evolvability of gene regulation in evolutionarily distant organisms. To facilitate comparison of Gcn5's role in the conserved stress responses of S. cerevisiae and $S$. pombe, it was necessary to further characterize the stress response role of Gcn5 in S. cerevisiae.

\section{Defects in the conserved HAT activity of Gcn5 cause stress sensitivity}

The HAT domain of Gcn 5 is the most conserved domain with a sequence homology of $67 \%$ between budding yeast and human [25]. To test whether Gen5 HAT activity is important for the stress response we tested the ability of plasmids expressing wild type or mutant gcn 5 alleles, containing triple alanine substitutions, to rescue the salt sensitivity of $g c n 5 \Delta$. The Gcn5-KQL and Gcn5-PKM substitution mutants have previously been shown to abolish HAT activity, while the Gcn5-PKE mutant maintains full catalytic activity [25]. The relative location of the mutated amino acid residues in the Gen 5 HAT domain is indicated in Fig. 1B. Expression of wild type Gen5 and Gcn5-PKE restores wild type growth levels during $\mathrm{KCl}$ and $\mathrm{CaCl}_{2}$ stress while strains expressing the Gcn5-PKM and Gcn5-KQL proteins with defective HAT activity display the same level of stress sensitivity as $g c n 5 \Delta$ (Fig. 1C). We conclude that the conserved HAT activity of Gcn5 is required for its role in stress responses.

\section{Identification of $\mathrm{KCl}$ stress response genes}

To study how Gcn5 is involved in the $\mathrm{KCl}$ stress response of $S$. cerevisiae, we first identified genes with changed expression levels during $\mathrm{KCl}$ adaptation in wild type cells. Fig. 2A shows that many genes show either increased or decreased expression in response to $\mathrm{KCl}$ (see also gene list in Additional file 2). The gene ontology terms most significantly associated with the sets of $\mathrm{KCl}$ induced and repressed genes are shown in Table 1 . The response to $\mathrm{KCl}$-stress conditions is complex but appears to include down-regulation of some general cellular functions (e.g. translation, amino acid biosynthesis) as well as induced expression of genes involved in stress adaptation (e.g. stress response and ion homeostasis). Down regulation of genes involved in protein synthesis during the response of $S$. cerevisiae to other stress conditions has been reported previously [26,27]. More than 30\% of genes regulated during $\mathrm{KCl}$ adaptation are general environmental stress response genes [28] (Fig. 2B). We found a similar proportion of common stress response genes among the $\mathrm{KCl}$ stress response genes of $S$. pombe, as described previously [22]. We also found similar gene ontology categories for $\mathrm{KCl}$-induced genes in both yeast species, such as response to stress/stimuli, oxidoreductase activity and carbohydrate metabolic process.

\section{Identification of $\mathrm{Gcn} 5$ dependent $\mathrm{KCl}$ stress response genes}

Next, we investigated the role of Gcn5 in the $\mathrm{KCl}$ stress response of $S$. cerevisiae. Comparison of the expression profiles for $g c n 5 \Delta$ cells in relation to wild type in the absence and presence of $\mathrm{KCl}$ showed that a significant number of genes become more dependent on Gcn5 under $\mathrm{KCl}$ stress conditions, as shown by the broader distribution of Gcn5-dependent effects in the presence of $\mathrm{KCl}$ (Fig. 3A). Interestingly, there is a fairly equal distribution between genes that require Gcn 5 for expression, consistent with its role as a coactivator, and genes that are directly or indirectly repressed by Gcn5. To define a group of $\mathrm{Gcn} 5$ dependent $\mathrm{KCl}$ responsive genes, we selected a group of 92 genes as summarised in Fig. 3B. Briefly, the group of 92 genes contains $\mathrm{KCl}-$ induced genes that show reduced expression in the gcn5 5 mutant during $\mathrm{KCl}$ stress (64 genes, see Additional file 3) as well as $\mathrm{KCl}$-repressed genes that show increased expression in $g c n 5 \Delta$ during $\mathrm{KCl}$ stress (28 genes, Additional file 3). The gene ontology categories most significantly associated with Gcn5 dependant $\mathrm{KCl}$ response genes (Table 2) include categories that would be expected to play an important role during adaptation to $\mathrm{KCl}$ stress conditions, such as ion homeostasis.

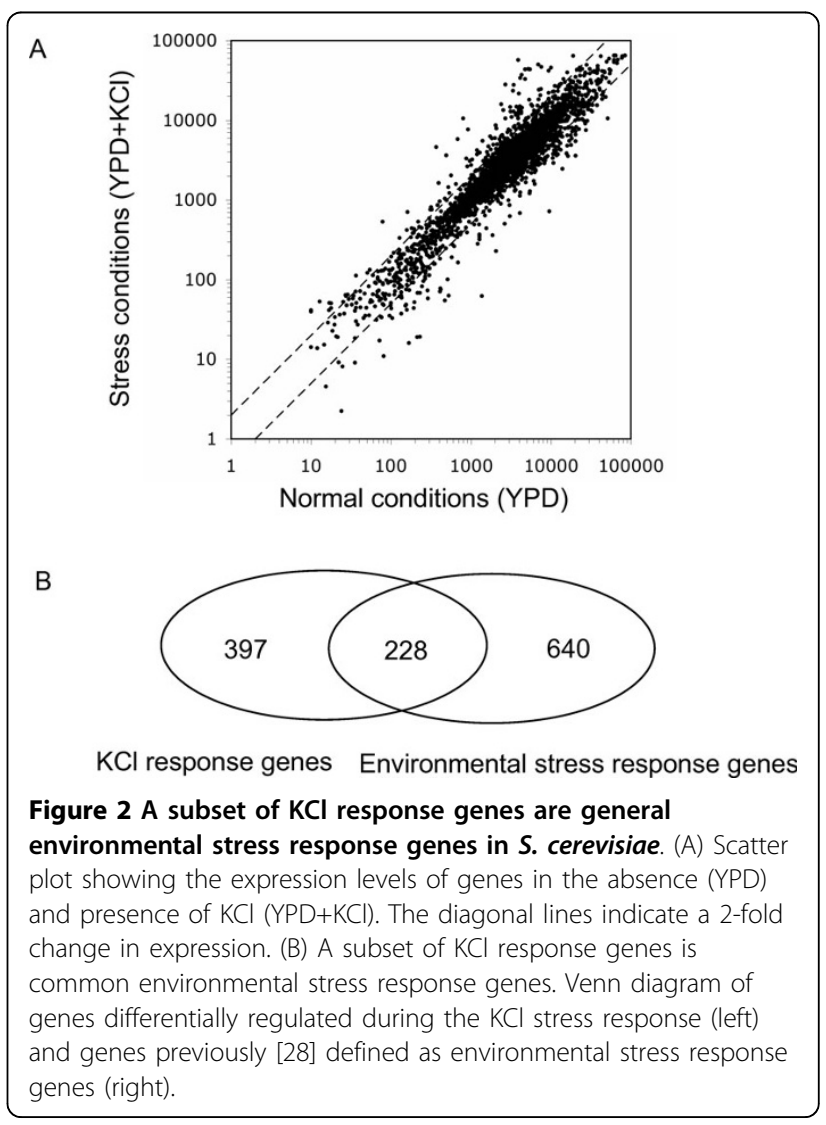


Table 1 Gene Ontology categories enriched in $\mathrm{KCl}$-responsive genes

\begin{tabular}{|c|c|c|c|}
\hline GO Category & Total genest & Changed genes* & $\mathrm{P}$ value\# \\
\hline \multicolumn{4}{|l|}{ KCl-induced genes (284) } \\
\hline Response to stress & 582 & 42 & 0.004 \\
\hline Oxidoreductase activity & 322 & 33 & $2.11 \mathrm{e}-5$ \\
\hline Carbohydrate metabolic process & 266 & 28 & $5.77 e-5$ \\
\hline Generation of precursor metabolites and energy & 180 & 18 & 0.002 \\
\hline Vacuole cell cycle-correlated morphology & 165 & 17 & 0.002 \\
\hline Cofactor metabolic process & 176 & 17 & 0.004 \\
\hline Ion homeostasis & 121 & 12 & 0.013 \\
\hline Glucose metabolic process & 88 & 11 & 0.003 \\
\hline \multicolumn{4}{|l|}{$\mathrm{KCl}-$ repressed genes (341) } \\
\hline Gene expression & 1438 & 150 & $2.81 \mathrm{e}-16$ \\
\hline Protein metabolic process & 1449 & 120 & $4.93 e-06$ \\
\hline Translation & 399 & 101 & $2.48 \mathrm{e}-41$ \\
\hline Structural constituent of ribosome & 221 & 80 & $4.804 \mathrm{e}-45$ \\
\hline Ribosome biogenesis and assembly & 356 & 70 & $5.11 \mathrm{e}-21$ \\
\hline Organic acid metabolic process & 386 & 51 & $1.28 \mathrm{e}-08$ \\
\hline Carboxylic acid metabolic process & 386 & 51 & $1.28 \mathrm{e}-08$ \\
\hline RNA binding & 476 & 50 & $2.04 \mathrm{e}-05$ \\
\hline Oxidoreductase activity & 322 & 32 & 0.001 \\
\hline Protein-RNA complex assembly & 140 & 29 & $1.08 \mathrm{e}-09$ \\
\hline Amino acid biosynthetic process & 134 & 23 & $2.11 \mathrm{e}-06$ \\
\hline Cofactor binding & 156 & 20 & 0.001 \\
\hline Lyase activity & 96 & 14 & 0.001 \\
\hline Glutamine family amino acid metabolic process & 61 & 11 & 0.001 \\
\hline Vitamin binding & 61 & 11 & 0.001 \\
\hline
\end{tabular}

† Total genes in the GO category.

*Number of $\mathrm{KCl}$ induced of reduced genes in the $\mathrm{GO}$ category.

\# $p$-value from a Fisher's Extract Test (one-sided), statistical considerations are fully discussed in [44].

\section{Characterization of direct Gcn5 target genes}

We validated several Gcn5-dependent $\mathrm{KCl}$ response genes by semi-quantitative PCR (Additional file 4A). This group includes a membrane transporter $V M R 1$ (YHL035C) and a heat-shock-response gene SSA4 (YER103W). The latter is consistent with a recent report that Gcn5 and Elp3 induced histone H3 acetylation is important for regulating Hsp70 expression [29]. Interestingly, we also confirmed that the FLO8 gene, which encodes a transcriptional regulator, is up-regulated in gcn5 5 (Fig. 4A, left panel). This is typical of many Gcn5 dependent genes and its dependence on Gcn5 for its stress-regulated repression is of interest because Gcn 5 is generally regarded as a transcriptional activator. One possibility is that up-regulation of FLO8 in gcn5 4 could be an indirect effect of the Gcn5 defect. To identify direct targets of Gcn5 we performed ChIP-on-chip experiments to identify the genome-wide localisation of myc-tagged Gcn5 using high resolution tiling arrays under the $\mathrm{KCl}$ stress conditions in which $\mathrm{Gcn} 5$ is required for FLO8 expression. This analysis showed reproducible interaction of Gcn5 with the FLO8 transcribed region under $\mathrm{KCl}$ stress conditions, suggesting direct repression of FLO8 expression by Gcn5 (Fig. 4A, middle panel). Direct association of Gcn5 with FLO8 was further confirmed by semi-quantitative PCR (Fig. $4 \mathrm{~A}$, right panel), The antibody used for precipitation of Gcn5-myc is specific because no signals were detected either in the absence of antibody or in a strain lacking the specific epitope tag in the presence of antibody (see Additional file 4B). We conclude that Gcn5 functions as a negative regulator for $F L O 8$ under $\mathrm{KCl}$ stress conditions. A repressive role of the Gcn5-containing SAGA complex has been reported previously. In S. cerevisiae, Gcn 5 is required for repression of $A R G 1$ on rich media where expression is not required but the role of Gcn5 switches on minimal media, where it is required for induction of $A R G 1$ [30]. A similar function of Gcn5 and the SAGA complex has been reported in $S$. pombe, in which Gcn 5 represses ste 11 and mei2, while another 
A

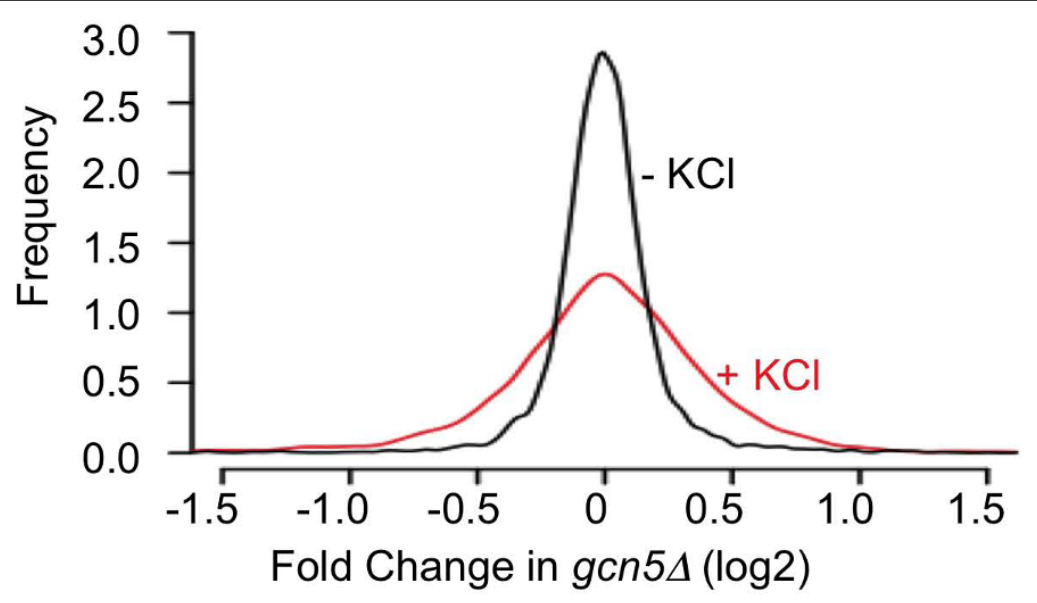

B

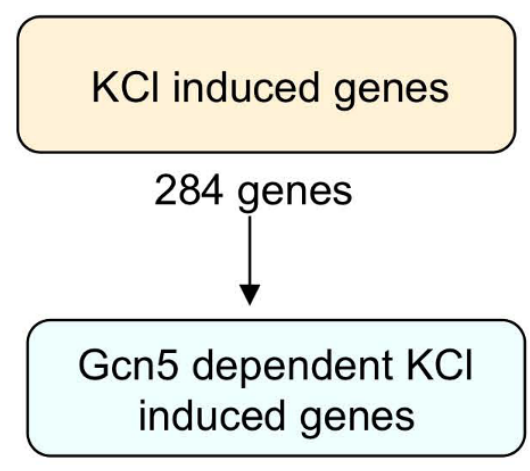

64 out of 284 genes
$\mathrm{KCl}$ repressed genes

341 genes

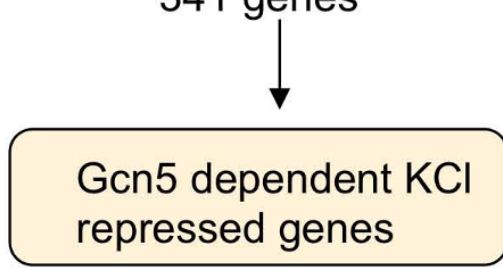

28 out of 341 genes
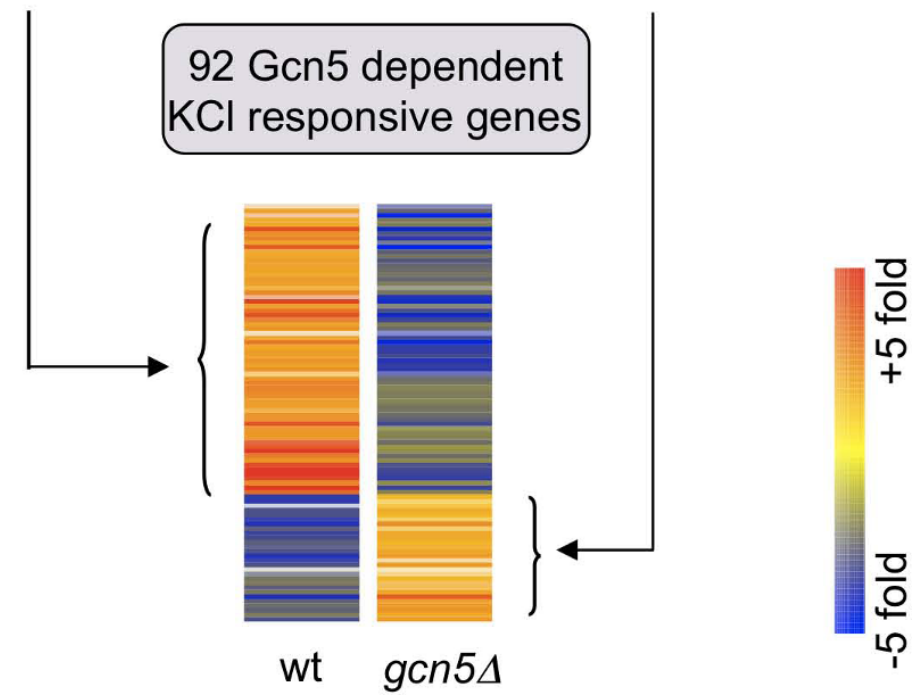

Figure 3 Identification of $\mathrm{Gcn} 5$ dependent $\mathrm{KCl}$ stress response genes in S. cerevisiae. (A) The Gcn5 dependency of global gene expression increases during $\mathrm{KCl}$ induced stress. Graph shows the frequency distribution ( $y$-axis, arbitrary units) of the Gcn5 dependency of genes ( $x$-axis) in the absence (black curve) and presence (red curve) of $\mathrm{KCl}$. The Gcn5 dependency for each gene is expressed as the ratio between the mean transcript level in the gcn5 $\Delta$ strain in relation to the mean transcript level in the wild type strain. (B) Diagram illustrating how Gcn5 dependent $\mathrm{KCl}$ response genes were defined. The selected genes are differentially regulated in wild type cells during $\mathrm{KCl}$ stress conditions in a fashion that requires $\mathrm{Gcn} 5$ when gcn5 $\Delta$ is compared to wild type during $\mathrm{KCl}$ adaptation. Selection criteria are described in the Methods section. The number for of genes in each group is indicated. The heat map shows the fold change values for these genes in the two data sets used to identify them, $+\mathrm{KCl} / \mathrm{KCl}(\mathrm{wt})$ and gcn5 $\Delta$ wild type $(g \mathrm{cn} 5 \Delta)$. 
Table 2 Gene Ontology categories enriched in $\mathrm{Gcn} 5$ dependant $\mathrm{KCl}$ response genes

\begin{tabular}{|c|c|c|c|}
\hline GO Category & Total genest & Changed genes* & $P$ value\# \\
\hline \multicolumn{4}{|l|}{ Gcn5 dependent $\mathrm{KCl}$ induced genes (64) } \\
\hline Catalytic activity & 2271 & 33 & 0.016 \\
\hline Oxidoreductase activity & 322 & 13 & $2.58 \mathrm{e}-5$ \\
\hline Cofactor binding & 156 & 7 & 0.001 \\
\hline Ion homeostasis & 121 & 6 & 0.002 \\
\hline Protein folding & 97 & 4 & 0.019 \\
\hline \multicolumn{4}{|l|}{ Gcn5 dependent $\mathrm{KCl}$-repressed genes (28) } \\
\hline Gene expression & 1438 & 14 & 0.004 \\
\hline Ribonucleoprotein complex biogenesis and assembly & 426 & 9 & 0.001 \\
\hline RNA processing & 448 & 6 & 0.019 \\
\hline Translation factor activity nucleic acid binding & 58 & 4 & $1.64 \mathrm{e}-4$ \\
\hline Iron ion binding & 106 & 4 & 0.002 \\
\hline Protein-RNA complex assembly & 140 & 4 & 0.004 \\
\hline Glutamine metabolic process & 21 & 3 & $1.3 e-4$ \\
\hline Flocculation & 11 & 2 & 0.001 \\
\hline
\end{tabular}

† Total genes in the GO category.

*Selection criteria see methods.

$\# p$-value from a Fisher's Extract Test (one-sided), statistical considerations are fully discussed in [44].

A

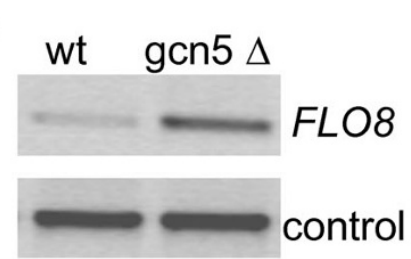

Expression
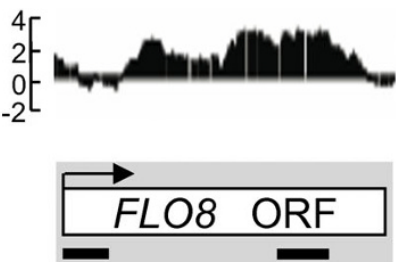

B

A

ChIP on chip
Input ChIP

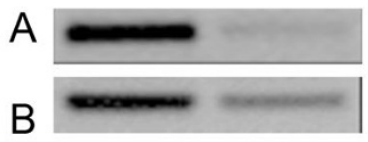

ChIP PCR

B

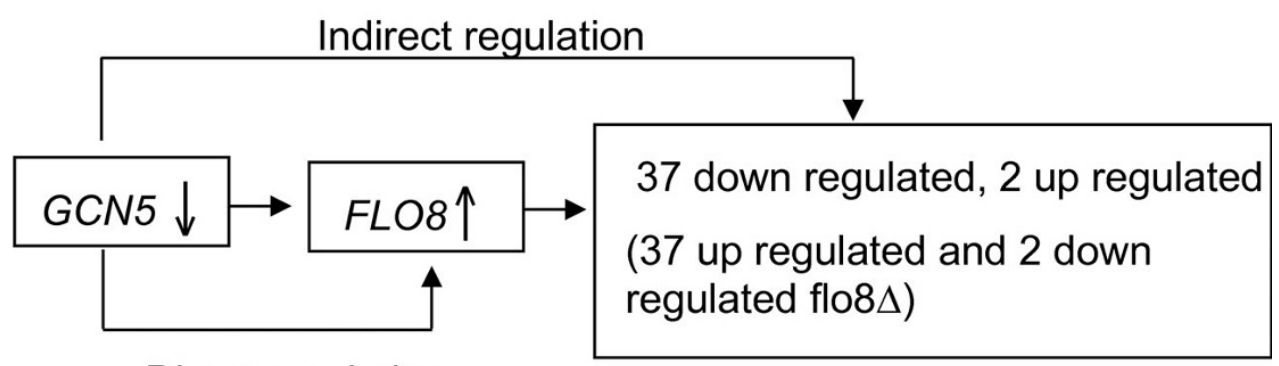

\section{Direct regulation}

Figure $4 \mathrm{Gcn} 5$ is associated with the transcribed region of the $\mathrm{FLO} 8$ gene and causes its repression during $\mathrm{KCl}$ stress. (A) Verification of FLO8 regulation and binding by Gcn5. The relative expression level of $F L O 8$ gene in wild type and gcn5 $\triangle$ cells under $\mathrm{KCl}$ stress conditions is shown (left panel) in relation to a control gene, YDL212W. Middle panel: tiling array data visualized by IGB showing relative enriched association of Gcn5-myc with FLO8 in immunoprecipiated material normalized against input material. The arrow shows the direction of transcription. A and $B$ represent the location of the amplified binding region and control region used for semi quantitative PCR for ChIP, Right panel: semi quantitative PCR validation of Gcn5 association with FLO8 showing the level of Gcn5-myc associated chromatin in relation to the input for regions $A$ and $B$ as designated in the middle panel. (B) Diagram showing how deletion of Gcn5 causes indirect regulation of FLO8 target genes. FLO8 $\triangle$ vs wild type microarray data are from ([32] GSE4654). 
SAGA subunit, Spt8 is required for their activation under inducing conditions [31].

As expected, we observed enriched association of Gcn5myc on many Gcn5-dependent genes. However, there are other Gcn5-dependent genes for which we do not observe enrichment of Gcn5. The detection of transcription factor genes, such as $F L O 8$, among the direct Gcn5 targets suggests that many of the indirect target genes might be regulated by such transcription factors. This is true for Flo8, where 39 of 44 Flo8 target genes identified previously [32] are dependent on Gcn5 in our study. Of these 39 genes, 37 were up-regulated and 2 were downregulated in the absence of FLO8. In gcn $5 \Delta$ cells, where FLO8 is up-regulated, these genes show the opposite regulatory patterns as would be expected if their Gcn 5 dependence is an indirect consequence of defects in the direct regulation of $F L O 8$ by Gen5 (Fig. 4B).

\section{Changes in the pattern of Gcn5 localization under stress conditions}

To analyse the genome-wide pattern of Gcn5 localisation as well as whether the localisation pattern changes upon $\mathrm{KCl}$ stress, we analysed the distribution of Gcn5 within genes in the absence and presence of $\mathrm{KCl}$ using average gene analysis. In this method, data for each gene is mapped onto a hypothetical gene of average length, thus allowing studies of average Gcn 5 distribution across a model gene for different groups of genes. First we examined the average localisation of Gcn 5 in sets of genes that are either up-regulated, down-regulated or unchanged in cells lacking Gcn5 (Fig. 5A). In the absence of $\mathrm{KCl}, \mathrm{Gcn} 5$ tends to be most abundant at or close to the promoter region (Fig. 5A left panel). This is consistent with previous data showing that Gen5 is localised predominately to promoter regions [33] as well as the known pattern of histone acetylation within genes $[34,35]$. In the presence of $\mathrm{KCl}$, the average localisation of Gen5 is predominantly within the transcribed region of genes (Fig. 5A right panel). The changed average distribution of Gcn 5 to transcribed regions during $\mathrm{KCl}$ stress conditions suggests a role of Gcn5 in the transcribed regions of genes under these conditions. Such a role has been suggested for Gen 5 on the GAL1 gene where it appears to play a transcription-coupled role in nucleosome eviction in the transcribed region [36] during induction of the gene by galactose. Recently we showed that Gen5 plays an important role in transcriptional elongation in S. pombe [20]. It is thus possible that an elongation-related role of Gen 5 may be conserved throughout the eukaryotes.

In S. pombe, Gcn5 tends to be preferentially located within the transcribed regions of highly expressed genes in the absence of stress [20]. To test whether this is so in $S$. cerevisiae we used average gene analysis to test the distribution of Gen 5 in sets of genes containing genes expressed at different levels (Fig. 5B). In the absence of stress Gcn 5 tends to be preferentially localised to highly expressed genes but it is not preferentially located in the transcribed region. However, during $\mathrm{KCl}$ stress the average localisation of Gcn5 is in the transcribed region but its preference for highly expressed genes under these conditions is marginal and may not be significant.

It was reported previously [36] that a very long coding region expressed from the GAL1 promoter was more sensitive to defects in Gen5 than the normal GAL1 gene, which has a shorter coding region. In particular, the level of RNA Polymerase II at the 3'-end of the long-gene coding region was sensitive to defects in Gcn5. This suggests that Gcn 5 might be particularly important for transcription of long genes in S. cerevisiae. To investigate this at the genome-wide level we used average gene analysis to study the distribution of Gcn5 on gene sets containing genes of different length, both in the absence and presence of $\mathrm{KCl}$ stress (Fig. 5C). In the absence of stress (Fig. 5C, left panel) the level of Gcn 5 at transcribed regions is inversely related to their length. This pattern is completely changed upon $\mathrm{KCl}$ stress, where there is a clear positive correlation between the levels of Gcn 5 in the transcribed regions and gene length (Fig. 5C, right panel).

The changes in the average distribution of Gcn5 upon $\mathrm{KCl}$ stress could be due to a shift in the location of Gen5 on individual genes or it could result from enhanced or depleted levels of Gcn5 on specific classes of genes that together make up the observed average localisation patterns. To distinguish between these possibilities, we used K-means clustering to identify the most clearly defined gene sets according to their average Gcn5 localisation pattern (Fig. 5D). Interestingly, we identified five gene clusters for which the average Gcn5 localisation pattern was representative of the majority of individual genes within the cluster (see Additional file 5). Comparison of the average Gcn 5 localisation for these clusters in the absence and presence of $\mathrm{KCl}$ showed differences that can account for the overall changes in average Gcn 5 localisation. Notably, Cluster 2, containing genes for which Gcn 5 tends to be located in the transcribed region, shows enhanced Gcn 5 association in response to stress. Furthermore, Cluster 3, containing genes with an even distribution of Gen5 throughout the gene, shows a strong reduction in the average level of Gcn 5 association. The changes in these two clusters are the main reasons for the apparent shift of Gcn5 to transcribed regions during stress. The remaining clusters show smaller changes in the average Gcn5 localisation pattern but the subtle changes that do occur also tend to specifically enhance the level of average Gcn5 association with transcribed regions. 


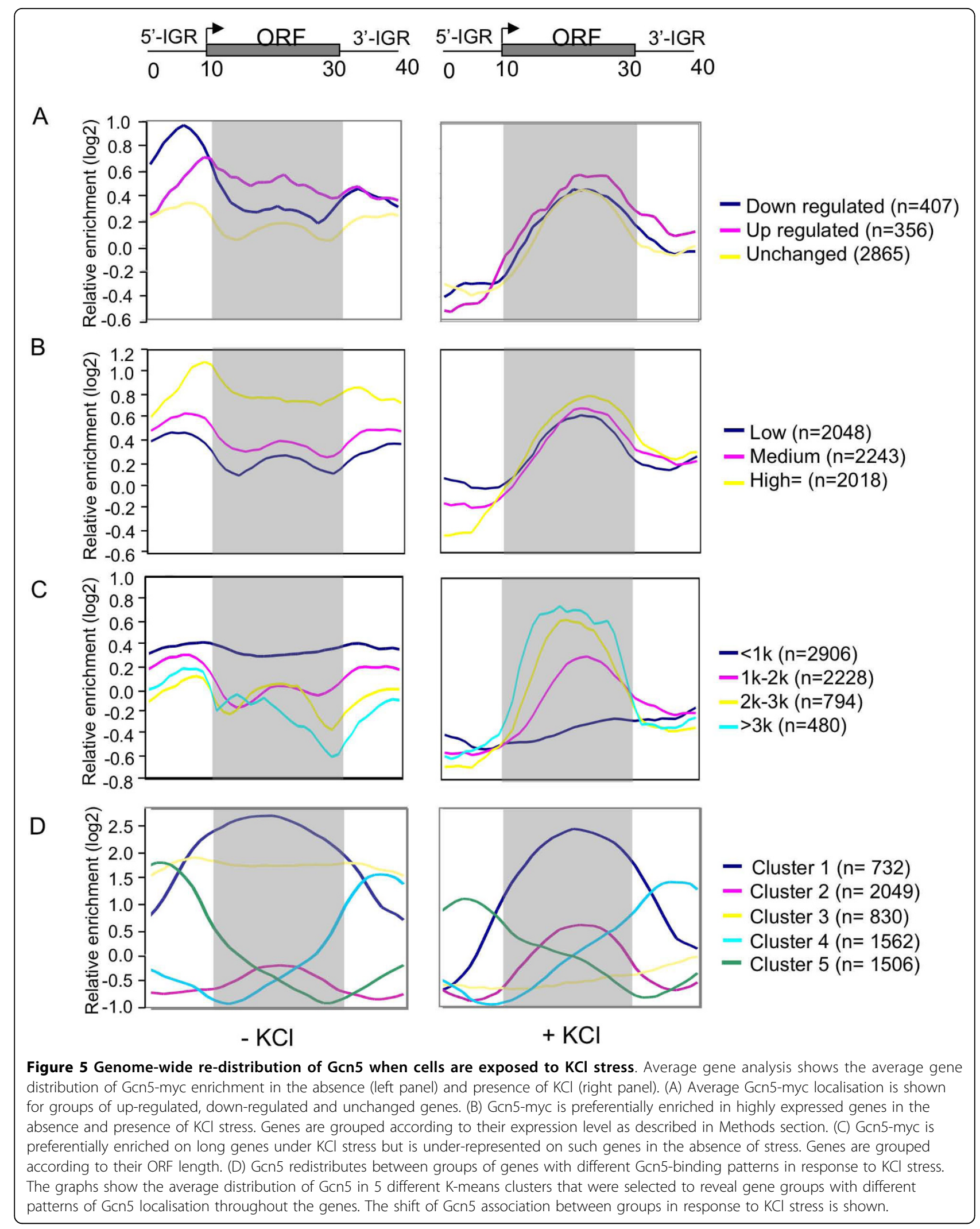


Strikingly, Cluster 2 is significantly enriched in long genes $(>2000 \mathrm{bp}, \mathrm{p}=1.31 \mathrm{E}-67)$; it contains 605 genes that are longer than $2000 \mathrm{bp}$, representing about half of all the genes within this length category. Cluster 3 is significantly enriched in short genes $(<500 \mathrm{bp}, \mathrm{p}=5.85 \mathrm{E}-$ 47) and includes 301 genes shorter than $500 \mathrm{bp}$. Thus, adaptation to $\mathrm{KCl}$ stress is associated with a net transfer of Gcn 5 from short genes, where it tends to be evenly distributed throughout genes, to long genes, in which it is predominantly localised within the transcribed region. Fig. 6 shows the re-distribution of Gen 5 on individual genes chosen from Clusters 2 and 3 in response to $\mathrm{KCl}$ mediated stress. The Gcn 5 redistribution between different genes thus accounts for the changes in average Gen5 localisation shown in Fig. 5C.

\section{Comparative analysis of gene regulation by Gen 5 during stress adaptation}

Since gene regulatory networks evolve rapidly (see Introduction), we did not expect Gcn5 to be involved in evolutionarily conserved functional applications even though it is a highly conserved protein at the structural and mechanistic levels. It was therefore of interest that Gcn 5 plays a role in adaptation to a common set of stress responses in evolutionarily divergent yeasts (Fig. 1A). To determine whether cross-species conservation is also seen at the level of Gcn5 target genes, we identified a set of Gcn5-dependent $\mathrm{KCl}$ response genes in S. pombe (123 genes, see Additional file 6) in previously published microarray data [22], using the same criteria as for S. cerevisiae data in this work. Gene Ontology analysis showed that several functional categories are enriched in $\mathrm{Gcn} 5$ dependent $\mathrm{KCl}$ response genes in both yeasts (e.g. transmembrane transporter activity, iron-ion binding, glutamate metabolic process). To compare whether orthologous pairs of genes are involved in Gcn 5 dependent adaptation to $\mathrm{KCl}$ stress in S. cerevisiae and $S$. pombe, we used unambiguous orthologous relationships that have been predicted for 2900 pairs of genes [37], which is about half of all S. cerevisiae genes. Comparison of the $92 \mathrm{Gcn} 5$ dependent $\mathrm{KCl}$ responsive genes in $S$. cerevisiae with the equivalent group of 123 genes identified in $S$. pombe revealed only 2 cases where both $S$. cerevisiae and $S$. pombe genes were members of an orthologous group, with the same regulation pattern (Fig. 7A). The extent of the overlap is at a level that would be expected by chance and may thus not be statistically significant. The two orthologous pairs are YDL171C-SPAB1E7.07, encoding a glutamate synthase, which has a function in detoxification, and YDR223W-SPAC22H10.11C, encoding a transcriptional corepressor, involved in the regulation of ribosomal protein gene transcription. It was formally possible that the $\mathrm{KCl}$ response could be so diverged between the two yeast species at the gene level, that there would be few or no co-regulated orthologous gene pairs that are potential targets for Gcn5. However, this appears not to be the case since there are many orthologous gene pairs that are regulated similarly in response to $\mathrm{KCl}$ stress in both S. cerevisiae and S. pombe (Additional file 7). We conclude that Gcn5 regulates divergent sets of $\mathrm{KCl}$ responsive genes in $S$. cerevisiae and $S$. pombe.

Finally, we wanted to find out if there is a similar change in the distribution pattern of Gen 5 when cells are subjected to stress conditions in the two yeasts. To allow comparison with the S. cerevisiae ChIP-onchip data described above we performed analogous ChIP-on-chip experiments to determine the localisation of Gcn5-myc in S. pombe during $\mathrm{KCl}$ stress adaptation. Equivalent data in the absence of stress have been published recently [20]. We selected groups of genes from $S$. cerevisiae and S. pombe that showed a level of Gcn5 enhancement greater than 2.6-fold ( $\log 2$ 1.4) in both the absence and presence of $\mathrm{KCl}$. The localisation pattern in S. cerevisiae differs between the two conditions consistent with the results in Fig. 5 (Fig. 7B upper panel), while the pattern for Gcn5-myc in S. pombe is similar under both conditions (Fig. 7B lower panel). The different behaviour of $S$. cerevisiae and $S$. pombe suggests that there are differences in the redistribution behaviour of Gcn5 between the two yeasts.

The results published here provide new insights regarding the role of coregulator proteins such as Gcn5 during physiological adaptation in the budding yeast, $S$. cerevisiae. The results also provide insights about how the enzymatically conserved Gcn 5 protein is utilized divergently in evolutionarily divergent organisms. Although Gen 5 plays a role in a set of stress responses that seems to be conserved across the large evolutionary distance between budding and fission yeasts, we find that Gcn5 is important for the expression of divergent sets of genes during adaptation to at least one of these stress conditions. This finding is consistent with existing findings that support the highly evolvable nature of gene regulation networks (see Introduction). The divergent requirement for Gcn 5 might thus be due to altered recruitment to target genes as a result of changes in the position or composition of transcription factor binding sites or changes in the repertoire of protein interaction partners available to transcription factor proteins. A recent comparative study provides a similar example between much more closely related species, $S$. cerevisiae and Candida glabrata [38]. The gene expression responses to oxidative stress are remarkably conserved 


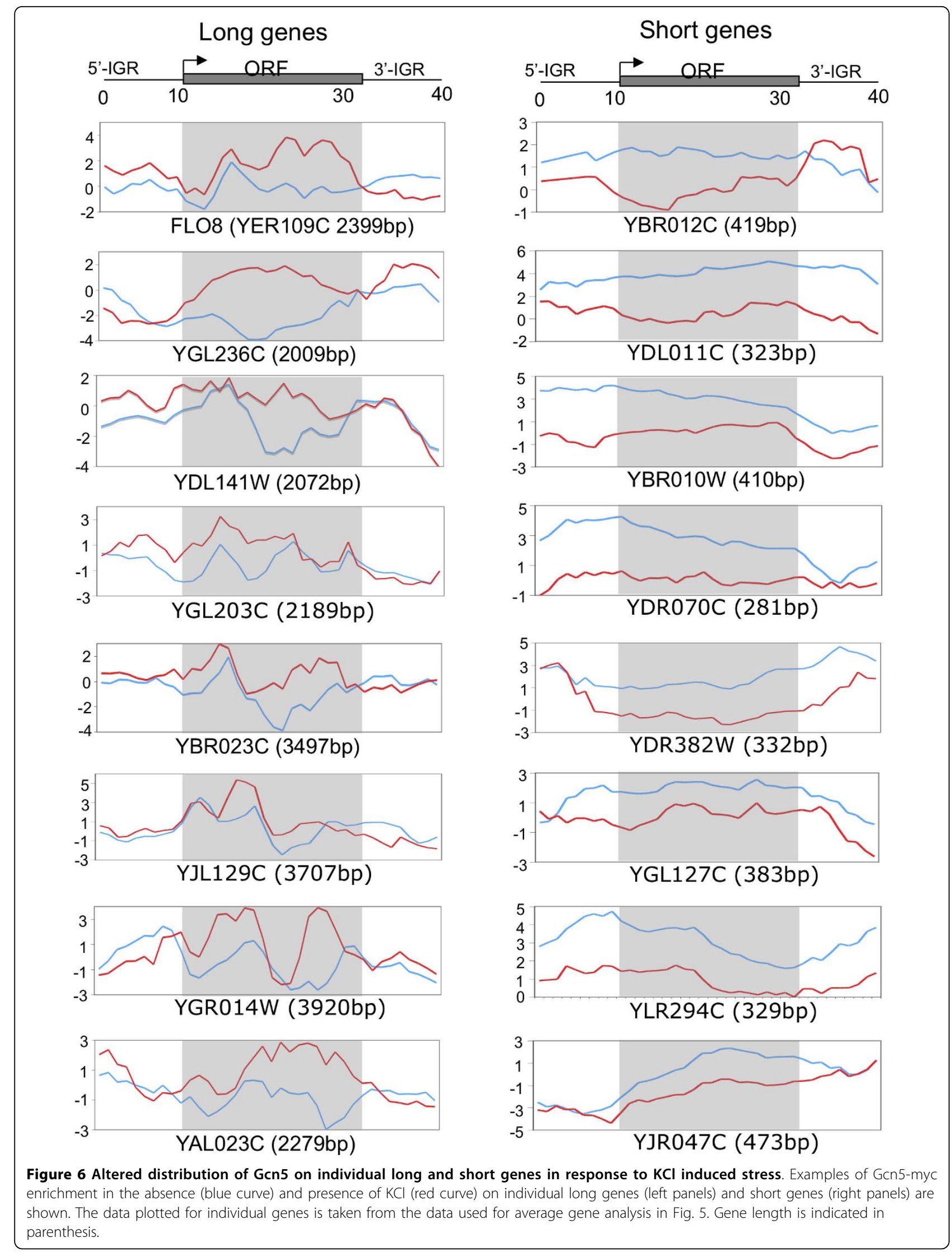


A

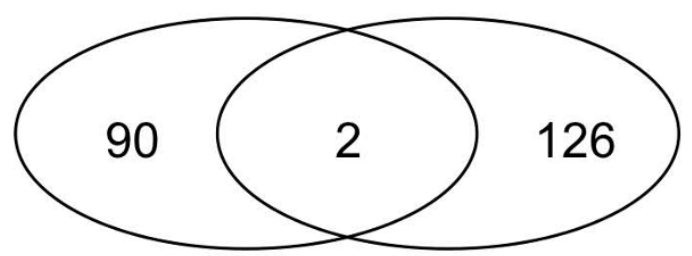

$\begin{array}{ll}\text { S. cerevisiae } & \text { S. pombe }\end{array}$

B
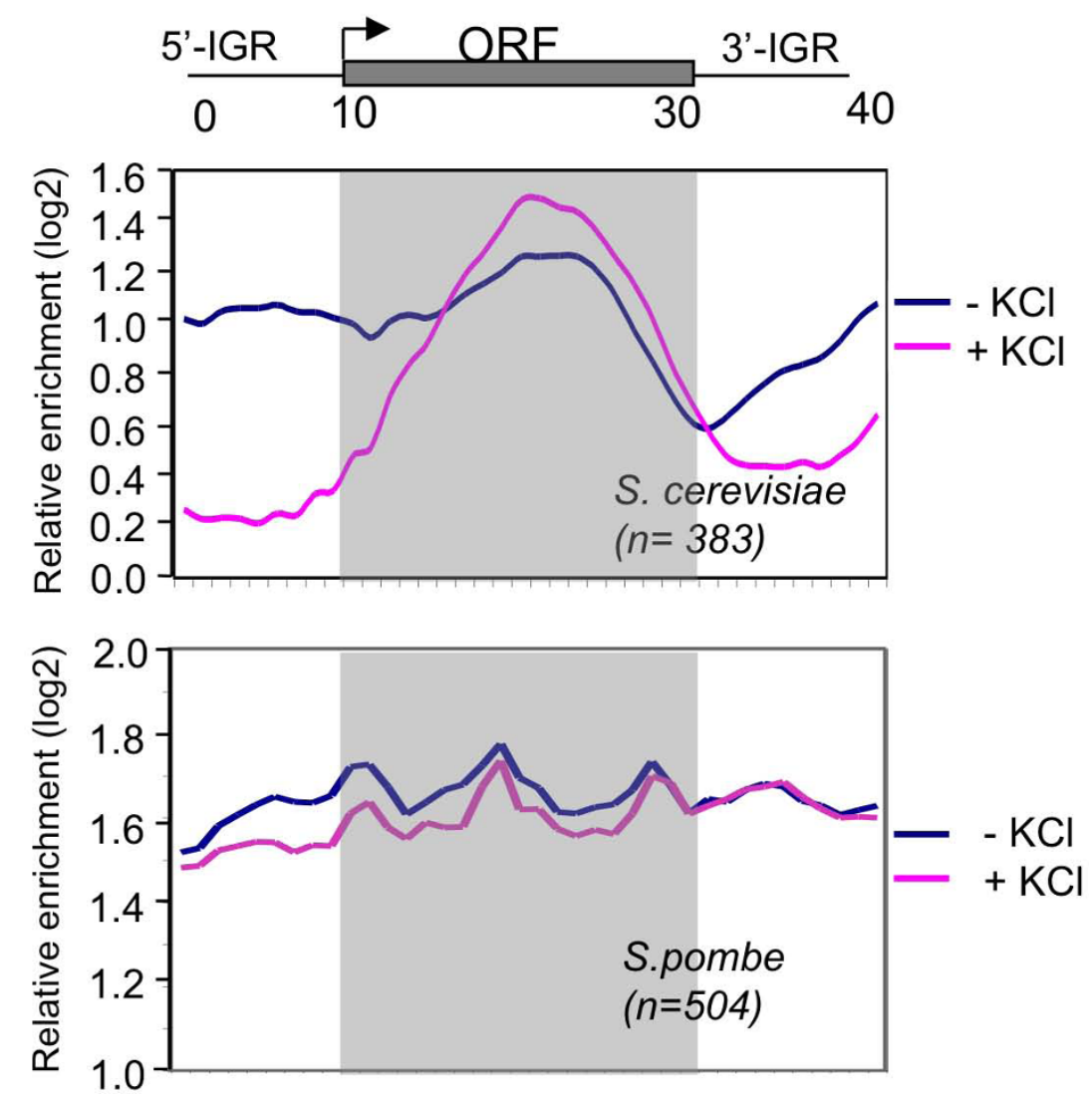

Figure $7 \mathrm{Gcn} 5$ differs in gene regulation and genome-wide localisation in S. cerevisiae and S. pombe during $\mathrm{KCl}$ stress adaptation. (A) $\mathrm{Gcn} 5$ regulates different sets of $\mathrm{KCl}$ responsive genes in $\mathrm{S}$. cerevisiae and S. pombe. The Venn diagram shows the relationship between gene sets identified in S. cerevisiae and S. pombe. (B) The altered Gcn5-myc gene distribution pattern between normal and $\mathrm{KCl}$-stress conditions in S. cerevisiae is not observed in S. pombe. The average gene distribution of Gcn5-myc is shown for genes that are associated with Gcn5 in both the absence and presence of $\mathrm{KCl}$ stress. The distribution of $\mathrm{Gcn} 5$ is shown for conditions in the absence (blue line) and presence of $\mathrm{KCl}$ (red line) for S. cerevisiae (average of 383 genes, upper panel) and S. pombe (average of 504 genes, lower panel), respectively.

between these two species, but the underlying regulatory networks differ. Each species appears to have different response motifs and the oxidative stress response transcription factor (Yap1p in S. cerevisiae and Cgap1p in C. glabrata) show clear differences in the way they "read" the cis-regulatory elements present in target promoters. Other examples of transcription networks, such as the regulation of S-phase related transcription in budding and fission yeasts by the MBF/SBF transcription factors, show a high level of evolutionary conservation between transcription factors and their target genes [39].

Gcn5 is a structurally and mechanistically conserved protein that functions by acetylating other proteins, notably histone $\mathrm{H} 3$, in a specific manner via its HAT 
domain or by specifically binding to acetylated histone $\mathrm{H} 3-\mathrm{K} 14$ residues via its Bromo domain. As mentioned above the difference in the identity of Gcn5-dependent stress genes in $S$. cerevisiae and $S$. pombe is most likely a result of divergence in the genes to which Gen5 is recruited in different yeasts, since gene regulation networks are known to evolve rapidly. Over the evolutionary distance separating $S$. cerevisiae and $S$. pombe we would not expect to find any conservation of biological function for the Gcn 5 protein. It is thus interesting to consider why Gcn5 function is required for a common set of stress responses in the different yeast species studied here. One possibility is that the evolution of Gcn 5 function is constrained by some aspect of its function, other than the target genes it regulates. For example, Gcn5, or perhaps other proteins in the SAGA complex, could be regulated by evolutionarily conserved stressspecific signaling pathways that modulate Gcn5 activity. This mechanism would require some stress genes to be Gcn 5 dependent but the identity of such genes could be different in different yeasts. Interestingly, there are known examples in which coregulators are direct targets for signaling pathways [40]. It is also possible that loss of Gcn5 recruitment to stress genes during evolution often creates growth defects during stress that tend to be suppressed by acquisition of Gen 5 recruitment by other genes involved in the same stress response, as a result of natural selection.

The results provide new insights into the mechanistic role played by Gcn 5 during stress adaptation, which appear to show both similarities and differences between $S$. cerevisiae and $S$. pombe. We provide further support for the direct role of Gcn5 as a repressor of many genes as has been suggested previously for both $S$. cerevisiae and $S$. pombe (discussed above). The re-distribution of Gcn 5 between genes during stress adaptation appears to be more dynamic in $S$. cerevisiae than in $S$. pombe, where the average Gcn5 localisation profiles are similar in the absence and presence of stress. The gene cluster (Cluster 2) showing enhanced association of Gcn5 in the transcribed region during stress adaptation contains about one third of all the genes. 145 of these genes are regulated during $\mathrm{KCl}$ adaptation and 199 are dependent on Gcn5. These gene sets are significantly enriched in long genes $(\mathrm{p}<0.0005)$. Therefore, the enhanced Gcn5 binding during $\mathrm{KCl}$ adaptation can account for some of the observed regulatory changes and Gcn5 may have a role in transcribed gene regions that is specific for long genes. The role of Gcn5 in transcribed regions is associated with the efficiency of transcriptional elongation in $S$. pombe. This may also be the case in $S$. cerevisiae but further studies are required to test this. Gcn 5 has been shown to be important for eviction of nucleosomes from the transcribed region of the GAL1 gene [36], which would be consistent with an elongation role in $S$. cerevisiae.

\section{Conclusions}

Gcn5 is an evolutionarily conserved protein that is required for a common set of stress responses across a highly divergent range of yeast species. These responses require the activity of the conserved HAT domain but are mediated by divergent sets of Gcn 5 dependent response genes in S. cerevisiae and S. pombe. Gcn5 is localised to $S$. cerevisiae genes that require its activity for their repression as well as for activation, thus providing support for the suggestion that Gcn 5 can function as a direct repressor of gene activity in addition to its characterized role as a transcriptional coactivator. Gcn5 localisation tends to shift from short genes to the transcribed regions of long genes during stress adaptation in $S$. cerevisiae. No such change is detectable in $S$. pombe. In the absence of stress Gcn5 is preferentially localised on highly expressed genes in $S$. cerevisiae, as previously reported for $S$. pombe.

\section{Methods}

\section{Strains, plasmids and growth conditions}

The yeast strains used in this study are listed in Table 3. The GCN5 deletion mutation in S. kluyveri, was made by first inserting PCR products (c. $500 \mathrm{bp}$ ) containing sequences up- and down-stream of the Gcn 5 coding sequence into pFA6a-KanMX6 at the BamHI and EcoRI sites, respectively. The primers used are listed in Additional file 8. A DNA fragment containing the KanMX cassette flanked by the up- and down-stream flanking regions was then amplified and transformed into the wild type strain of S. kluyveri, Y090. Kanamycin resistant colonies in which the Gcn5 coding region was replaced by the KanMX cassette were selected on plates containing G418 (50 mg/ml) and confirmed by PCR.

S. cerevisiae strains expressing Gcn 5 derivatives containing substitution mutations in the HAT domain were made by transferring mutant alleles of GCN5 from existing plasmids, PKE-pRS306, PKM-pRS306, KQL-pRS306, YG5pRS414 [25], to pRS316 [41]. The PvuI fragment from the donor plasmid containing GCN5 replaced the equivalent fragment from pRS316 that lacked GCN5. The new plasmids, PKE-pRS316, PKM-pRS316, KQL-pRS316, YG5pRS316 (control containing wild type GCN5) were transformed into gcn $5 \Delta$ deletion strain BY7285 and BY17285 and transformants were selected on SD-ura plates.

S. cerevisiae and $S$. kluyveri strains were cultivated in YPD medium (1\% yeast extract, $2 \%$ bacto peptone and $2 \%$ glucose) and $S$. pombe strains were cultivated in YEA medium $(0.5 \%$ yeast extract, $3 \%$ glucose, $0.2 \%$ casamino acids with $100 \mathrm{mg} / \mathrm{L}$ of adenine, uracil and leucine, respectively) All the spotting assays were 
Table 3 Yeast strains used in this study

\begin{tabular}{llll}
\hline Strain name & Yeast species & Genotype & Source \\
\hline Fy368 & S. pombe & h-, leu-32, ura4-D18, ade6-M210 & Ekwall K \\
Hu799 & S. pombe & $h$-, gcn5::KAN-MX, leu-32, ura4-D18, ade6-M210 & [22] \\
By4741 & S. cerevisiae & MATa, his3-1, leu2-0, met15-0, ura3-0 & Murén E \\
By7285 & S. cerevisiae & By4741, gcn5::KAN-MX & Murén E \\
By4742 & S. cerevisiae & MAT $\alpha$, his3-1, leu2-0, lys2-0, ura3-0 & Murén E \\
By17285 & S. cerevisiae & By4742, gcn5::KAN-MX & Murén E \\
Y090 & S. Kluyveri & MATo thr & Piškur J \\
HuY090 g & S. Kluyveri & Y090 gcn5::KAN-MX & this work \\
BQS1350 & S. cerevisiae & By4742 Gcn5-MYC13-KanMX6 & {$[17]$} \\
Hu2020 & S. pombe & h-, gcn5-myc::KanMX6, leu 1-32, ura4-D18, ade6-M210 & {$[20]$} \\
\hline
\end{tabular}

† Total genes in the GO category.

*Selection criteria see methods.

\# $p$-value from a Fisher's Extract Test (one-sided), statistical considerations are fully discussed in [44].

performed by spotting 5 -fold serial dilutions of cells on rich medium supplemented with different stress-inducing compounds as shown in Fig. 1A and Fig. 1C. Concentrations of $\mathrm{KCl}, \mathrm{CaCl}_{2}$, Calcoflour White, $\mathrm{MnCl}_{2}$, and caffeine for S. cerevisiae and S. kluyveri were $1 \mathrm{M}, 0.25$ $\mathrm{M}, 20 \mathrm{ug} / \mathrm{ml}, 4 \mathrm{mM}$ and $6 \mathrm{mM}$, respectively. The equivalent concentrations for $S$. pombe, were $1 \mathrm{M}, 0.1$ $\mathrm{M}, 2 \mathrm{mg} / \mathrm{ml}, 2 \mathrm{mM}$ and $8 \mathrm{mM}$ respectively. The growth incubation temperature was $25^{\circ} \mathrm{C}$ for $S$. cerevisiae and $S$. kluyveri and $30^{\circ} \mathrm{C}$ for $S$. pombe. For other stress conditions see Additional File 1.

\section{Gene expression profiling}

S. cerevisiae wild type and $g c n 5 \Delta$ strains were compared by expression profiling under exponential growth conditions at $25^{\circ} \mathrm{C}$. To determine the effect of $\mathrm{KCl}$ stress on gene expression, cells were treated as described in [22]. For each condition, at least two biological replicates were used. An equivalent number of replicates were analyzed using each of the two possible $\mathrm{Cy} 3 / \mathrm{Cy} 5$ dye orientations on double spotted microarray slides (Eurogentec SA Seraing, Belgium) and the results were used to calculate the mean fold change value for each gene for each condition tested. RNA extraction, probe labelling and hybridization were performed as previously described [42]. Slides were scanned using a Bio-rad VersArray ChipReader and quantified with Imagene 4.2 software. All the primary data were normalized by Lowess normalization using GeneSpring software. Regulated genes were defined as genes for which the fold changes exceeded a level equivalent to one standard deviation about the overall population mean and for which the change was statistically significant $(\mathrm{p}<0.05)$. The significance of gene expression changes was assessed using Student's t-test to determine the probability that mean fold change values differ from a ratio of one. The null hypothesis tested was that there is no difference.
Unchanged genes were defined as genes having a fold change value less than 1.2. All gene expression profiling data analyzed in this study is available at Gene Expression Omnibus (GEO) http://www.ncbi.nlm.nih.gov/projects/ geo under accession number: SuperSeriesGSE 16556/Subset series GSE5218.

\section{ChIP-on-chip microarray}

Two biological replicates are used to study enriched binding sites of Gcn5-myc under normal conditions and $1 \mathrm{M}$ $\mathrm{KCl}$ treatment conditions in S. cerevisiae and S. pombe. The ChIP-on chip experiments were carried out as in [20] except the differences in material in culture medium $(S$. cerevisiae: YPD, S. pombe: YES) and array used (GeneChip S. cerevisiae Tiling 1.0R, Genechip S. pombe tiling 1.0FR). The data are available at GEO under accession number: SuperSeriesGSE 16556/Subset series GSE16514.

\section{ChIP-on-chip data analysis}

Raw data from Affymetrix (CEL format) were analyzed using Model-based Analysis for Tiling-array (MAT) software [43] with a bandwidth of 250 in order to identify regions enriched in Gcn5. Visualization of data was performed using Affymetrix integrated genome Browser (IGB). Average gene analysis was done as in Johnsson et al [20]. ChIP-on-chip data of normal conditions and $\mathrm{KCl}$ stress conditions were normalized in order to have the same overall standard deviation and mean value. Gene with different expression levels were assigned into three groups by the mean signal intensities of at least three biological replicates of cDNA wild type cells on double spotted cDNA microarray slides (from SuperSeriesGSE 16556/Subset series GSE5218). K-means cluster analysis was performed using GeneSpring software. The number of clusters and iterations was 5 and 100 respectively. Standard correlation was used as the similarity measure. 


\section{Verification of gene expression and CHIP-on-chip data with semi-quantitative $P C R$}

To verify the gene expression profiling results, cell treatment and RNA extraction were as previously described $[22,42]$. Reverse transcription of RNA was carried out using reagents from Fermentas (Cat. No. K1611) according to the manufacturers instructions, followed by PCR. To verify the enriched binding of Gcn5-Myc from CHIP-on-chip data, chromatin immunoprecipitation was carried out as described above without the amplification step. Semi quantitative PCR was used to compare immuniprecipitated material for binding and non-binding regions (indicated in the Fig. $4 \mathrm{~A}$ as fragment $\mathrm{A}$ and B) in relation to input (chromatin extract before IP). The primers used are shown in Additional file 8.

\section{Gene ontology analysis}

GoMiner http://discover.nci.nih.gov/gominer/ was used to find gene ontology (GO) terms that are significantly enriched in selected sets of genes with different expression patterns in relation to the frequency of their occurrence in the set of all genes $(p \leq 0.02)$. $p$-values were not corrected for multiple hypothesis testing. The statistical analysis performed by the GoMiner algorithm is fully discussed elsewhere [44].

Additional file 1: Conditions tested by spotting assays of wild type and $g c n 5 \Delta$ mutants in the three yeast species. A. Additional stress conditions, which are: $\mathrm{LiCl}, \mathrm{MgCl}_{2}, \mathrm{NaCl}$ sorbitol, ethanol, elevated temperature and SDS. B. Table showing the mean colony size $( \pm S D)$, for each strain in each condition, the size of gcn5 $\Delta$ colonies relative to wild type (also plotted in part C), as well as a $p$-value showing the level of significance of size differences between mutant and wild type colonies.

Additional file 2: $\mathrm{KCl}$ response genes in S. cerevisiae. Gene list for up and down regulated genes under $\mathrm{KCl}$ stress response in $\mathrm{S}$. cerevisiae with their ratio changes.

Additional file 3: $\mathrm{Gcn} 5$ dependent $\mathrm{KCl}$ responsive genes in $\mathrm{S}$. cerevisiae. Gene list for $\mathrm{G} \mathrm{cn} 5$ dependent $\mathrm{KCl}$ response genes in $\mathrm{S}$. cerevisiae showing their expression pattern and annotation.

Additional file 4: Verification of $\mathrm{Gcn} 5$ dependent $\mathrm{KCl}$ response genes by semi-quantitative PCR and negative control experiments for ChIP specificity. Genes validated include: FLO8 (YER109C), VMR1 (YHL035C), HSP70 (YER103W) and control (YDL212W).

Additional file 5: Gcn5 localisation pattern on genes within the $5 \mathrm{~K}$ means clusters. Gcn 5 localisation pattern on genes within the $5 \mathrm{~K}$ means clusters studied in Fig. 5D in the absence and presence of $\mathrm{KCl}$.

Additional file 6: $\mathrm{Gcn} 5$ dependent $\mathrm{KCl}$ responsive genes in $\mathrm{S}$. pombe. Gene list for $\mathrm{Gcn} 5$ dependent $\mathrm{KCl}$ response genes with their expression pattern and annotation in S. pombe.

Additional file 7: Conserved and diverged regulation pattern of $\mathrm{KCl}$ response genes between $S$. cerevisiae and S. pombe. Hierarchical cluster analysis to compare the expression changes of $\mathrm{KCl}$ regulated genes in S. cerevisiae with the changes for their orthologs in S. pombe during $\mathrm{KCl}$ adaptation.

Additional file 8: PCR primers used in this study. The list of PCR primers used in this study.

\section{Acknowledgements}

We thank Shelley Berger, Eve Murén, Jure Piškur and Vicent Tordera's lab for providing plasmids and strains. We thank the Bioinformatics and Expression Analysis Core Facility at Karolinska Institute for help with the CHIP-chip tiling array study and Helmi Siltala for technical assistance. We appreciate help with the computer set up for tiling array analysis from Jürgen Hench and for comments to the manuscript from Yann Betrand. We thank Rachel Berkson for critical reading of the manuscript. AW is supported by grants from the Swedish Research Council, the Swedish Cancer Society and the European Commission (QLK3-CT-200000174).

\section{Author details}

${ }^{1}$ School of Life Sciences, Södertörn University, Huddinge, SE-141 89, Sweden. ${ }^{2}$ Center for Biosciences, Department of Biosciences and Nutrition, Karolinska Institute, Huddinge, SE-141 83, Sweden.

\section{Authors' contributions}

YX-F designed the experiments and performed the phenotypic studies and most micorarray experiments, as well as HAT functional study and PCR confirmation of regulated genes. She analysed the data, interpreted the results and wrote the manuscript. AJ performed part of microarray experiments and contributed to the writing process. DB, JH and TB provided assistence with programming and analysis of tiling array data. AW conceived the idea, supervised the research project and helped with manuscript writing. All authors read and approved the final manuscript.

Received: 17 June 2009 Accepted: 25 March 2010

Published: 25 March 2010

\section{References}

1. Prud'homme B, Gompel N, Carroll SB: Emerging principles of regulatory evolution. Proc Natl Acad Sci USA 2007, 104(Suppl 1):8605-8612.

2. Wray GA: The evolutionary significance of cis-regulatory mutations. Nat Rev Genet 2007, 8(3):206-216.

3. Wray GA, Hahn MW, Abouheif E, Balhoff JP, Pizer M, Rockman MV, Romano LA: The evolution of transcriptional regulation in eukaryotes. Mol Biol Evol 2003, 20(9):1377-1419.

4. Beskow A, Wright AP: Comparative analysis of regulatory transcription factors in Schizosaccharomyces pombe and budding yeasts. Yeast 2006, 23(13):929-935.

5. Kummerfeld SK, Teichmann SA: DBD: a transcription factor prediction database. Nucleic Acids Res 2006, 34 Database: D74-81.

6. Ranea JA, Buchan DW, Thornton JM, Orengo CA: Evolution of protein superfamilies and bacterial genome size. J Mol Biol 2004, 336(4):871-887.

7. van Nimwegen E: Scaling laws in the functional content of genomes. Trends Genet 2003, 19(9):479-484.

8. Bürglin TR: Homeodomain Proteins. Encyclopedia of Molecular Cell Biology and Molecular Medicine. Wiley-VCH Verlag GmbH \& Co., Weinheim 2005.

9. Burglin TR: The homeobox genes of Encephalitozoon cuniculi (Microsporidia) reveal a putative mating-type locus. Dev Genes Evol 2003, 213(1):50-52.

10. Mattick JS: A new paradigm for developmental biology. J Exp Biol 2007, 210(Pt 9):1526-1547.

11. Lynch VJ, Wagner GP: Resurrecting the role of transcription factor change in developmental evolution. Evolution 2008, 62(9):2131-2154.

12. Wagner $\mathrm{GP}$, Lynch $\mathrm{VJ}$ : The gene regulatory logic of transcription factor evolution. Trends Ecol Evol 2008, 23(7):377-385.

13. Brownell JE, Zhou J, Ranalli T, Kobayashi R, Edmondson DG, Roth SY, Allis CD: Tetrahymena histone acetyltransferase A: a homolog to yeast Gcn5p linking histone acetylation to gene activation. Cell 1996, 84(6):843-851.

14. Grant PA, Duggan L, Cote J, Roberts SM, Brownell JE, Candau R, Ohba R Owen-Hughes T, Allis CD, Winston F, et al: Yeast Gen5 functions in two multisubunit complexes to acetylate nucleosomal histones: characterization of an Ada complex and the SAGA (Spt/Ada) complex. Genes Dev 1997, 11(13):1640-1650. 
15. Grant PA, Eberharter A, John S, Cook RG, Turner BM, Workman JL: Expanded lysine acetylation specificity of Gcn5 in native complexes. J Biol Chem 1999, 274(9):5895-5900.

16. Helmlinger DMS, Villén J, Gygi SP, Bähler J, Winston F: The S. pombe SAGA complex controls the switch from proliferation to sexual differentiation through the opposing roles of its subunits Gcn5 and Spt8. Genes Dev 2008, 22(22):3184-3195.

17. Rosaleny LE, Ruiz-Garcia AB, Garcia-Martinez J, Perez-Ortin JE, Tordera V: The Sas $3 p$ and Gcn $5 p$ histone acetyltransferases are recruited to similar genes. Genome Biol 2007, 8(6):R119.

18. Utley RT, Ikeda K, Grant PA, Cote J, Steger DJ, Eberharter A, John S, Workman JL: Transcriptional activators direct histone acetyltransferase complexes to nucleosomes. Nature 1998, 394(6692):498-502

19. Bhaumik SR, Raha T, Aiello DP, Green MR: In vivo target of a transcriptional activator revealed by fluorescence resonance energy transfer. Genes Dev 2004, 18(3):333-343.

20. Johnsson A, Durand-Dubief M, Xue-Franzen $Y$, Ronnerblad M, Ekwall K, Wright A: HAT-HDAC interplay modulates global histone H3K14 acetylation in gene-coding regions during stress. EMBO Rep 2009, 10(9):1009-1014

21. Wang L, Mizzen C, Ying C, Candau R, Barlev N, Brownell J, Allis CD, Berger SL: Histone acetyltransferase activity is conserved between yeast and human GCN5 and is required for complementation of growth and transcriptional activation. Mol Cell Biol 1997, 17(1):519-527.

22. Johnsson A, Xue-Franzen Y, Lundin M, Wright AP: Stress-specific role of fission yeast Gcn 5 histone acetyltransferase in programming a subset of stress response genes. Eukaryot Cell 2006, 5(8):1337-1346.

23. Huisinga KL, Pugh BF: A genome-wide housekeeping role for TFIID and a highly regulated stress-related role for SAGA in Saccharomyces cerevisiae. Mol Cell 2004, 13(4):573-585.

24. Dujon B, Sherman D, Fischer G, Durrens P, Casaregola S, Lafontaine I, De Montigny J, Marck C, Neuveglise C, Talla E, et al: Genome evolution in yeasts. Nature 2004, 430(6995):35-44.

25. Wang L, Liu L, Berger SL: Critical residues for histone acetylation by Gcn5, functioning in Ada and SAGA complexes, are also required for transcriptional function in vivo. Genes Dev 1998, 12(5):640-653.

26. Teige $M$, Scheikl E, Reiser $V$, Ruis H, Ammerer G: Rck2, a member of the calmodulin-protein kinase family, links protein synthesis to high osmolarity MAP kinase signaling in budding yeast. Proc Natl Acad Sci USA 2001, 98(10):5625-5630.

27. Uesono $Y$, Toh EA: Transient inhibition of translation initiation by osmotic stress. J Biol Chem 2002, 277(16):13848-13855.

28. Gasch AP, Spellman PT, Kao CM, Carmel-Harel O, Eisen MB, Storz G, Botstein D, Brown PO: Genomic expression programs in the response of yeast cells to environmental changes. Mol Biol Cell 2000, 11(12):4241-4257.

29. Han Q, Lu J, Duan J, Su D, Hou X, Li F, Wang X, Huang B: Gen5- and Elp3induced histone $\mathrm{H} 3$ acetylation regulates hsp70 gene transcription in yeast. Biochem J 2008, 409(3):779-788.

30. Ricci AR, Genereaux J, Brandl CJ: Components of the SAGA histone acetyltransferase complex are required for repressed transcription of ARG1 in rich medium. Mol Cell Biol 2002, 22(12):4033-4042.

31. Helmlinger D, Marguerat S, Villen J, Gygi SP, Bahler J, Winston F: The S. pombe SAGA complex controls the switch from proliferation to sexual differentiation through the opposing roles of its subunits Gcn5 and Spt8. Genes Dev 2008, 22(22):3184-3195.

32. Hu Z, Killion PJ, Iyer VR: Genetic reconstruction of a functional transcriptional regulatory network. Nat Genet 2007, 39(5):683-687.

33. Robert F, Pokholok DK, Hannett NM, Rinaldi NJ, Chandy M, Rolfe A, Workman JL, Gifford DK, Young RA: Global position and recruitment of HATs and HDACs in the yeast genome. Mol Cell 2004, 16(2):199-209.

34. Millar CB, Grunstein M: Genome-wide patterns of histone modifications in yeast. Nat Rev Mol Cell Biol 2006, 7(9):657-666.

35. Li B, Carey M, Workman JL: The role of chromatin during transcription. Cell 2007, 128(4):707-719.

36. Govind CK, Zhang F, Qiu H, Hofmeyer K, Hinnebusch AG: Gen5 promotes acetylation, eviction, and methylation of nucleosomes in transcribed coding regions. $\mathrm{Mol}$ Cell 2007, 25(1):31-42.

37. Wood V: Comparative Genomics Using Fungi as Models, Topic in Current Genetics, Volumn15, Schizosaccharomyces pombe comparative genomics; from sequence to systems. Springer 2006, 15.
38. Lelandais G, Tanty V, Geneix C, Etchebest C, Jacq C, Devaux F: Genome adaptation to chemical stress: clues from comparative transcriptomics in Saccharomyces cerevisiae and Candida glabrata. Genome Biol 2008, 9(11): R164.

39. Oliva A, Rosebrock A, Ferrezuelo F, Pyne S, Chen H, Skiena S, Futcher B, Leatherwood J: The cell cycle-regulated genes of Schizosaccharomyces pombe. PLoS Biol 2005, 3(7):e225.

40. Bjorklund S, Gustafsson CM: The yeast Mediator complex and its regulation. Trends Biochem Sci 2005, 30(5):240-244

41. Sikorski RS, Hieter P: A system of shuttle vectors and yeast host strains designed for efficient manipulation of DNA in Saccharomyces cerevisiae. Genetics 1989, 122(1):19-27.

42. Xue $Y$, Haas SA, Brino L, Gusnanto A, Reimers $M$, Talibi D, Vingron $M$, Ekwall K, Wright AP: A DNA microarray for fission yeast: minimal changes in global gene expression after temperature shift. Yeast 2004, 21(1):25-39.

43. Johnson WE, Li W, Meyer CA, Gottardo R, Carroll JS, Brown M, Liu XS: Model-based analysis of tiling-arrays for ChIP-chip. Proc Natl Acad Sci USA 2006, 103(33):12457-12462.

44. Zeeberg BR, Feng W, Wang G, Wang MD, Fojo AT, Sunshine M, Narasimhan S, Kane DW, Reinhold WC, Lababidi S, et al: GoMiner: a resource for biological interpretation of genomic and proteomic data. Genome Biol 2003, 4(4):R28.

doi:10.1186/1471-2164-11-200

Cite this article as: Xue-Franzén et al:: Genome-wide characterisation of the Gcn5 histone acetyltransferase in budding yeast during stress adaptation reveals evolutionarily conserved and diverged roles. BMC Genomics 2010 11:200.

\section{Submit your next manuscript to BioMed Central and take full advantage of:}

- Convenient online submission

- Thorough peer review

- No space constraints or color figure charges

- Immediate publication on acceptance

- Inclusion in PubMed, CAS, Scopus and Google Scholar

- Research which is freely available for redistribution

Submit your manuscript at www.biomedcentral.com/submit
C) Biomed Central 